\title{
Publisher's Note: “Polymer-specific effects of bulk relaxation and stringlike correlated motion in the dynamics of a supercooled polymer melt" [J. Chem. Phys. 119, 5290 (2003)]
}

\author{
M. Aichele \\ Institut Charles Sadron, 6 rue Boussingault, 67083 Strasbourg, France \\ and Institut für Physik, Johannes Gutenberg-Universität, 55099 Mainz, Germany \\ Y. Gebremichael \\ Department of Chemical Engineering, University of Michigan, Ann Arbor, Michigan 48109 \\ and Chemical Physics Program, Institute for Physical Sciences and Technology, University of Maryland, \\ College Park, Maryland 20742 \\ F. W. Starra) \\ Center for Theoretical and Computational Materials Science and Polymers Division, \\ National Institute of Standards and Technology, Gaithersburg, Maryland 20899 \\ J. Baschnagel $\left.\right|^{\text {b) }}$ \\ Institut Charles Sadron, 6 rue Boussingault, 67083 Strasbourg, France \\ S. C. Glotzer ${ }^{\text {c) }}$ \\ Departments of Chemical Engineering and Materials Science and Engineering, University of Michigan, \\ Ann Arbor, Michigan 48109
}

[DOI: $10.1063 / 1.1690246]$

This article was originally published with an incorrect version of Fig. 8 on p. 5298. AIP apologizes for this error; the correct version of Fig. 8 appears below. All online versions of the article have been corrected.

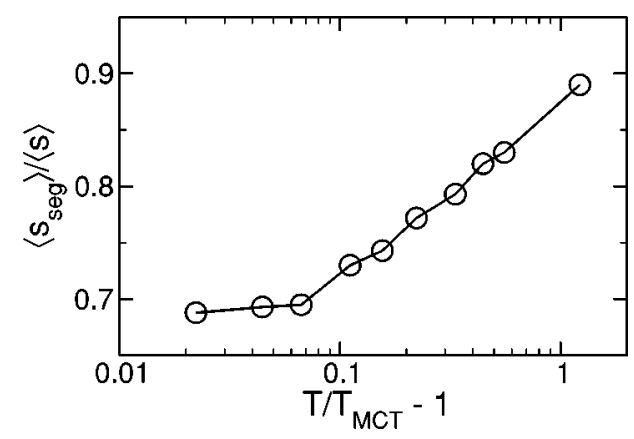

FIG. 8. Temperature dependence of the ratio of $\left\langle s_{\mathrm{seg}}\left(t_{\mathrm{str}}^{\max }\right)\right\rangle$ and $\left\langle s\left(t_{\mathrm{str}}^{\max }\right)\right\rangle$. $t_{\text {str }}^{\max }$ is the peak time of $\left\langle s_{\text {seg }}\right\rangle$ and $\langle s\rangle$ at different temperatures. $T_{\text {MCT }}$ $=0.45$.

\footnotetext{
a) Present address: Department of Physics, Wesleyan University, Middletown, CT 06459.

b) Author to whom correspondence should be addressed. Electronic mail: baschnag@ics.u-strasbg.fr

${ }^{c}$ Author to whom correspondence should be addressed. Electronic mail: sglotzer@umich.edu
} 